\title{
A WEAK VECTOR-VALUED BANACH-STONE THEOREM
}

\author{
LEANDRO CANDIDO AND ELÓI MEDINA GALEGO
}

(Communicated by Thomas Schlumprecht)

\begin{abstract}
For a locally compact Hausdorff space $X$ and a Banach space $E$, we denote by $C_{0}(X, E)$ the space of $E$-valued continuous functions on $X$ which vanish at infinity, endowed with the supremum norm. In the spirit of the classical Banach-Stone theorem, we prove that if $C_{0}(X, E)$ is isomorphic to $C_{0}(Y, E)$, where $E$ has non-trivial cotype and such that $E$ is separable or $E^{*}$ has the Radon-Nikodým property, then either $X$ and $Y$ are finite or $X$ and $Y$ have the same cardinality. In other words, we obtain a vector-valued extension of a 1978 B. Cengiz result, the scalar case $E=\mathbb{R}$ or $E=\mathbb{C}$.
\end{abstract}

\section{INTRODUCTION}

We follow the standard notation and terminology for measure theory and Banach space theory that can be found in 9,11 and 16 . If $E$ is the real or complex scalar field, as usual, the space $C_{0}(X, E)$ will be denoted by $C_{0}(X)$. In the case where $X$ is compact, these spaces will be designated by $C(X, E)$ and $C(X)$, respectively. If there is a linear isomorphism $T$ from the Banach space $E$ onto the Banach space $F$, we will write, in short, $E \sim F$. Moreover, to emphasize that $\left\|T^{-1}\right\|\|T\|<\lambda$ for some $1<\lambda<+\infty$, we will also write $E \stackrel{\lambda}{\sim} F$. The cardinality of a set $A$ will be denoted by $|A|$.

The classical Banach-Stone theorem states that if $C_{0}(X)$ and $C_{0}(Y)$ are isometrically isomorphic, then $X$ and $Y$ are homeomorphic (in short, $X \approx Y$ ); see [2] and [21. This theorem has been extended in different ways; see for instance [15]. We stress that in [1] and [6] this theorem was independently strengthened as follows.

Theorem 1.1. Let $X$ and $Y$ be locally compact Hausdorff spaces. Then

$$
C_{0}(X) \stackrel{2}{\sim} C_{0}(Y) \Longrightarrow X \approx Y .
$$

In [4], Cambern showed that 2 is the best constant for the above theorem; see also [8]. Moreover, in [7, without the bound-two hypothesis, Cengiz proved the following weak version of Theorem 1.1 .

Theorem 1.2. Let $X$ and $Y$ be locally compact Hausdorff spaces. Then

$$
C_{0}(X) \sim C_{0}(Y) \Longrightarrow|X|=|Y| \text {. }
$$

The Banach-Stone theorem has also been generalized for the real vector-valued continuous functions. In this setting, the furthest-reaching result is the following one, due to Behrends and Cambern [3].

Received by the editors October 28, 2011 and, in revised form, December 26, 2011.

2010 Mathematics Subject Classification. Primary 46B03, 46B25; Secondary 46E27, 46E40.

Key words and phrases. Banach-Stone theorem.

The first author was supported by CNPq, process number 142423/2011-4.

(C) 2013 American Mathematical Society 
Theorem 1.3. Suppose that $E$ is a uniformly non-square space. Then there exists $1<\lambda \leq 2$ such that for every locally compact Hausdorff space $X$ and $Y$ we have

$$
C_{0}(X, E) \stackrel{\lambda}{\sim} C_{0}(Y, E) \Longrightarrow X \approx Y .
$$

Recall that a Banach space $E$ is said to be uniformly non-square if there is a $\delta>0$ such that there do not exist $x$ and $y$ in the the unit ball of $E$ for which

$$
\left\|\frac{1}{2}(x+y)\right\|>1-\delta \text { and }\left\|\frac{1}{2}(x-y)\right\|>1-\delta .
$$

Theorems 1.2 and 1.3 naturally lead to the following question.

Problem 1.4. Suppose that $E$ is a uniformly non-square Banach space and $X$ and $Y$ are locally compact Hausdorff spaces such that

$$
C_{0}(X, E) \sim C_{0}(Y, E) .
$$

What can be said about the cardinalities of $X$ and $Y$ ?

The principal purpose of the present paper is to answer completely the above question. In an attempt to solve Problem 1.4 we have obtained a more general result concerning the isomorphisms between $C_{0}(X, E)$ spaces. Indeed, Enflo [12] proved that if $E$ is a uniformly non-square Banach space, then $E$ can be renormed to an equivalent uniformly convex Banach space. In particular, $E$ has non-trivial cotype [10, Theorem 14.1, p. 283]; that is, it has cotype $q$ for some $2 \leq q<\infty$.

Recall that a Banach space $E \neq\{0\}$ has cotype $2 \leq q<\infty$ if there is a constant $\kappa>0$ such that no matter how we select finitely many vectors $v_{1}, v_{2}, \ldots, v_{n}$ from $E$,

$$
\left(\sum_{i=1}^{n}\left\|v_{i}\right\|^{q}\right)^{\frac{1}{q}} \leq \kappa\left(\int_{0}^{1}\left\|\sum_{i=1}^{n} r_{i}(t) v_{i}\right\|^{2} d t\right)^{\frac{1}{2}},
$$

where $r_{i}:[0,1] \rightarrow \mathbb{R}$ denote the Rademacher functions, defined by setting

$$
r_{i}(t)=\operatorname{sign}\left(\sin 2^{i} \pi t\right) .
$$

Thus, inspired by [7] we prove the following vector-valued extension of Theorem 1.2 .

Theorem 1.5. Let $E$ be a Banach space having non-trivial cotype. If $E^{*}$ has the Radon-Nikodým property or $E$ is separable, then for every locally compact Hausdorff spaces $X$ and $Y$ we have

$$
C_{0}(X, E) \sim C_{0}(Y, E) \Longrightarrow|X||Y|<\aleph_{0} \text { or }|X|=|Y| .
$$

Observe that Theorem 1.5 is also a weak version of Theorem 1.3 and provides an immediate solution to Problem 1.4. Indeed, it suffices to recall that every uniformly convex space $E$ is reflexive [16, p. 31] and therefore $E^{*}$ has the Radon-Nikodým property [9, Corollary 13, p. 76].

Notice also that Theorem 1.5 covers the cases $E=l_{1}$ and $E=l_{p}(\Gamma)$, where $\Gamma$ is an arbitrary set and $1<p<\infty$. We do not know whether the statement of this theorem is also true for the remaining case $E=l_{1}(\Gamma)$, where $\Gamma$ is an uncountable set. Finally, notice that the classical Milutin Theorem [18, Theorem 21.5.10] shows that we cannot remove the non-trivial cotype hypothesis in Theorem 1.5 in the case 
where $E$ is separable. Indeed, for every infinite countable compact Hausdorff $K$ we have

$$
C(K, C([0,1])) \sim C(K \times[0,1]) \sim C([0,1] \times[0,1]) \sim C([0,1], C([0,1])) .
$$

Moreover, since for every uncountable set $\Gamma, l_{1}(\Gamma)$ has the Radon-Nikodým property [9, Corollary 8, p. 83] and

$$
C_{0}\left(\mathbb{N}, C_{0}(\Gamma)\right) \sim C_{0}(\Gamma) \sim C_{0}\left(\Gamma, C_{0}(\Gamma)\right)
$$

holds, it follows that the non-trivial cotype hypothesis in Theorem 1.5 cannot also be removed in the case where $E^{*}$ has the Radon-Nikodým property.

The remainder of this paper is organized as follows. In Section 2, we state some results on Banach spaces having non-trivial cotype which are necessary to prove Theorem 1.5 (Proposition 2.1 and Lemma 2.2). In Section 3 , we recall a RadonNikodým type vector measure theorem (Theorem 3.1) and also prove a simple result (Proposition 3.3) which allows us to provide a unified proof of Theorem 1.5] in its distinct cases: $E^{*}$ has the Radon-Nikodým property or $E$ is separable.

In order to prove Theorem [1.5, we will work with some convenient lowersemicontinuous functions associated to bounded linear operators from $C_{0}(X, E)$ to $C_{0}(Y, E)$. So, in Section 4, we introduce these functions (Lemma 4.1) and recall Cengiz's result concerning certain lower-semicontinuous functions and real measures (Proposition 4.2). Finally, in Section 5, we prove Theorem 1.5.

\section{On $C_{0}(X, E)$ SPACES With $E$ HAVING NON-TRIVIAL COTYPe}

In this section we state some auxiliary results on Banach spaces having nontrivial cotype. For an $E$-valued measure $\mu,|\mu|$ denotes the variation of $\mu$ and the symbol rcabv $(X, E)$ comprises the Banach space of all regular, countably additive, Borel, bounded variation measures, endowed with the variation norm. As usual, when $E$ is a scalar field, this space is simply denoted by $r c a b v(X)$. Throughout we will use the Singer Representation Theorem. That is, there exists an isometric isomorphism between $C_{0}(X, E)^{*}$ and $r c a b v\left(X, E^{*}\right)$ such that every linear functional $\varphi$ and the corresponding measure $\mu$ are related by

$$
\langle\varphi, f\rangle=\int f d \mu, \quad f \in C_{0}(X, E),
$$

where the integral is termed the immediate integral of Dinculeanu [11, p. 11]; see [5. p. 2], [17] and also [14] when $X$ is a compact space.

Proposition 2.1. Let $E$ be a Banach space having non-trivial cotype and $X$ and $Y$ be locally compact Hausdorff spaces. Suppose that $T$ is an isomorphism of $C_{0}(X, E)$ into $C_{0}(Y, E)$ and set

$$
X_{y}=\left\{x \in X:\left\|T^{*}\left(\varphi \delta_{y}\right)(\{x\})\right\|>0 \text { for some } \varphi \in S_{E^{*}}\right\},
$$

where $\delta_{y}$ stands for the unit point mass at $y$ and $S_{E^{*}}$ denotes the unit sphere of $E^{*}$. Then

(a) $X_{y}$ is countable for every $y \in Y$.

(b) If $Y$ is infinite and for every $x \in X$ there exists a $y \in Y$ such that $x \in X_{y}$, then $|X| \leq|Y|$. 
Proof. (a) We assume that for some $y \in Y, X_{y}$ is uncountable and show that this assumption leads to a contradiction.

Hence there exist some $0<\epsilon<1$ such that

$$
\left\{x \in X:\left\|T^{*}\left(\varphi \delta_{y}\right)(\{x\})\right\|>\epsilon \text { for some } \varphi \in S_{E^{*}}\right\}
$$

is infinite.

Since $E$ has cotype $q$, for some $2 \leq q<\infty$, by (1.1) there exists a constant $Q>0$ in such a way that no matter how we select finitely many vectors $v_{1}, v_{2}, \ldots, v_{m} \in E$, if $0<\delta \leq\left\|v_{i}\right\|$ for each $1 \leq i \leq m$, there is an appropriate choice of scalars $r_{i}= \pm 1$ such that

$$
\left\|\sum_{i=1}^{m} r_{i} v_{i}\right\| \geq \delta Q \sqrt[q]{m}
$$

Pick $n \in \mathbb{N}$ satisfying $\epsilon Q \sqrt[q]{n}>2\|T\|$. Also fix distinct points $x_{1}, \ldots, x_{n} \in X$ and $\varphi_{1}, \ldots, \varphi_{n} \in S_{E^{*}}$ such that

$$
\left\|T^{*}\left(\varphi_{i} \delta_{y}\right)\left(\left\{x_{i}\right\}\right)\right\|>\epsilon, 1 \leq i \leq n .
$$

Thus, there are $v_{1}, \ldots, v_{n}$ in the unit ball of $E$ in such a way that

$$
\left\langle T^{*}\left(\varphi_{i} \delta_{y}\right)\left(\left\{x_{i}\right\}\right), v_{i}\right\rangle>\epsilon, 1 \leq i \leq n .
$$

Since $T^{*}\left(\varphi_{i} \delta_{y}\right)$ is regular for each $1 \leq i \leq n$, we can take $U_{1}, \ldots, U_{n}$ mutually disjoint open neighborhoods of $x_{1}, \ldots, x_{n}$, respectively, verifying

$$
\left|T^{*}\left(\varphi_{i} \delta_{y}\right)\right|\left(U_{i} \backslash\left\{x_{i}\right\}\right) \leq \frac{\epsilon}{2} .
$$

By the Urysohn Lemma [13, Theorem 1.5.11, p. 41], we can find $h_{i} \in C_{0}(X)$ with $0 \leq h_{i} \leq 1, h_{i}\left(x_{i}\right)=1$ and $h_{i}(x)=0$ if $x \in X \backslash U_{i}$. Define $f_{i} \in C_{0}(X, E)$ by $f_{i}=v_{i} . h_{i}$. By (2.2) we have

$$
\begin{aligned}
\left\|\left(T f_{i}\right)(y)\right\| & \geq\left|\left\langle\varphi_{i},\left(T f_{i}\right)(y)\right\rangle\right|=\left|\int f_{i} d T^{*}\left(\varphi_{i} \delta_{y}\right)\right| \\
& \geq\left|\left\langle T^{*}\left(\varphi_{i} \delta_{y}\right)\left(\left\{x_{i}\right\}\right), v_{i}\right\rangle\right|-\left|\int f_{i} d T^{*}\left(\varphi_{i} \delta_{y}\right)-\left\langle T^{*}\left(\varphi_{i} \delta_{y}\right)\left(\left\{x_{i}\right\}\right), v_{i}\right\rangle\right| \\
& >\epsilon-\left|T^{*}\left(\varphi_{i} \delta_{y}\right)\right|\left(U_{i} \backslash\left\{x_{i}\right\}\right) \geq \frac{\epsilon}{2} .
\end{aligned}
$$

According to (2.1) there is an appropriate choice of scalars $r_{i}= \pm 1$ such that

$$
\left\|\sum_{i=1}^{n} r_{i}\left(T f_{i}\right)(y)\right\| \geq \frac{\epsilon Q \sqrt[q]{n}}{2} .
$$

On the other hand, since $U_{i} \cap U_{j}=\emptyset$ if $i \neq j$ and $\left\|f_{i}\right\| \leq 1$ for each $1 \leq i \leq n$, we have

$$
\left\|\sum_{i=1}^{n} r_{i} f_{i}\right\| \leq 1
$$

Therefore, by (2.3) and the choice of $\epsilon$ we conclude that

$$
\|T\| \geq\left\|T\left(\sum_{i=1}^{n} r_{i} f_{i}\right)\right\| \geq\left\|T\left(\sum_{i=1}^{n} r_{i} f_{i}\right)(y)\right\|>\|T\|,
$$

which is the desired contradiction. 
(b) It follows by our hypothesis that

$$
X=\bigcup_{y \in Y} X_{y}
$$

By Proposition 2.1 (a), $X_{y}$ is countable for all $y \in Y$. Hence $|X| \leq|Y|$.

Note that Theorem [1.5] in the case where $X$ and $Y$ are finite is an immediate consequence of the following result.

Lemma 2.2. Let $E$ be a Banach space having non-trivial cotype and $X$ be a finite Hausdorff topological space. Then for every locally compact Hausdorff space $Y$ we have

$$
C_{0}(X, E) \sim C_{0}(Y, E) \Longrightarrow|Y|<\aleph_{0}
$$

Proof. There exists some $n \in \mathbb{N}$ such that

$$
E^{n} \sim C_{0}(X, E) \sim C_{0}(Y, E) .
$$

Then $Y$ must be finite. Otherwise, it is well known that $C_{0}(Y, E)$ contains a copy of $c_{0}$. Therefore, by (2.4) and [19, Theorem 1], $E$ contains a copy of $c_{0}$, which is absurd, because $E$ has non-trivial cotype.

\section{ON WEAKLY*-MEASURABLE FUNCTIONS}

This section is devoted to presenting certain weakly*-measurable functions which will be used later. First of all, recall that, given a Banach space $E$ and a locally compact Hausdorff space $Y$, a function $\gamma: Y \rightarrow E^{*}$ is said to be simple whenever its image is finite and $f^{-1}(v)$ is a Borel set for every $v \in E^{*}$. Moreover, $\gamma$ is measurable if there exists a sequence of simple functions converging pointwise to $\gamma$. Finally, $\gamma$ is weakly*-measurable if for every $v \in E$ the numerical function $y \mapsto\langle\gamma(y), v\rangle$ is measurable [11, Definition 5, p. 4, and Definition 16, p. 8].

We will need the following Radon-Nikodým type vector measure theorem [11, Theorem 34, p. 37].

Theorem 3.1. Let $E$ be a separable Banach space, $Y$ a locally compact Hausdorff space and $\mu \in \operatorname{rcabv}\left(Y, E^{*}\right)$. Then there exists a weakly*-measurable function $\gamma$ : $Y \rightarrow E^{*}$ such that

(a) $\|\gamma(y)\|=1$ for every $y \in Y$.

(b) The application $y \mapsto\langle\gamma(y), f(y)\rangle$ is measurable and

$$
\int f d \mu=\int\langle\gamma(y), f(y)\rangle d|\mu|(y)
$$

for every function $f: Y \rightarrow E$ which is a uniform limit of a sequence of E-valued simple functions.

Remark 3.2. Notice that if $E^{*}$ has the Radon-Nikodým property [9, Definition 3, p. 61], then it follows that we can choose $\gamma$ measurable, satisfying the same statements of Theorem 3.1 .

Proposition 3.3. Let $E$ be a Banach space, $Y$ a locally compact Hausdorff space, $\mu \in \operatorname{rcabv}(Y)$ and $\gamma: Y \rightarrow E^{*}$ a function such that $\|\gamma(y)\|=1$ for every $y \in Y$. Suppose that

(a) $E$ is separable and $\gamma$ is weakly ${ }^{*}$-measurable or

(b) $\gamma$ is measurable. 
Then for every $\epsilon>0$ there is a compact $K \subset Y$ such that $|\mu|(Y \backslash K)<\epsilon$ and the restriction of $\gamma$ to $K$ is continuous with respect to the weak ${ }^{*}$-topology of $E^{*}$.

Proof. (a) Consider $S_{E^{*}}$ endowed with its weak*-topology. Let $\left\{v_{n}: n \in \mathbb{N}\right\}$ be a dense sequence in $S_{E^{*}}$ and set

$$
\mathrm{d}(\psi, \varphi)=\sum_{n=1}^{+\infty} \frac{1}{2^{n}}\left|\left\langle\psi, v_{n}\right\rangle-\left\langle\varphi, v_{n}\right\rangle\right|, \quad \psi, \varphi \in S_{E^{*}}
$$

It follows that d is a metric inducing the topology of $S_{E^{*}}$.

Pick $\epsilon>0$. For each $n \in \mathbb{N}$, since the range $\gamma(Y) \subseteq S_{E^{*}}$, we can cover $\gamma(Y)$ by a finite collection of open balls $\left\{U_{1}, \ldots, U_{r_{n}}\right\}$ of radius $\frac{1}{2 n}$.

For each $1 \leq i \leq r_{n}$, suppose that $U_{i}=\left\{\psi \in S_{E^{*}}: \mathrm{d}\left(\psi, \varphi_{i}\right)<\frac{1}{2 n}\right\}$ and define $\eta_{i}: Y \rightarrow \mathbb{R}$ by $\eta_{i}(y)=\mathrm{d}\left(\gamma(y), \varphi_{i}\right)$. Since $\gamma$ is weakly*-measurable, $\eta_{i}$ is measurable because it is a pointwise limit of measurable functions. By

$$
\eta_{i}^{-1}\left(\left[0, \frac{1}{2 n}[)=\gamma^{-1}\left(U_{i}\right),\right.\right.
$$

we infer that $\gamma^{-1}\left(U_{i}\right)$ is a Borel set for every $1 \leq i \leq r_{n}$.

Next, for each $1 \leq i \leq r_{n}$ we define

$$
A_{i}=U_{i} \backslash \bigcup_{j<i} U_{j} \text { and } B_{i}=\gamma^{-1}\left(A_{i}\right)
$$

Therefore $B_{i}$ is a Borel set. By the regularity of $|\mu|$, we can find a compact $J_{i} \subset B_{i}$ such that

$$
|\mu|\left(B_{i} \backslash J_{i}\right) \leq \frac{\epsilon}{r_{n} 2^{n}} .
$$

For each $1 \leq i \leq r_{n}$, let $y_{i} \in J_{i}$ and define

$$
\gamma_{n}=\sum_{i=1}^{r_{n}} \gamma\left(y_{i}\right) \chi_{J_{i}}
$$

Since $J_{1}, \ldots, J_{r_{n}}$ are mutually disjoint, for every $y \in J_{1} \cup \ldots \cup J_{r_{n}}$,

$$
\mathrm{d}\left(\gamma(y), \gamma_{n}(y)\right)<\frac{1}{n} .
$$

Set $K_{n}=J_{1} \cup \ldots \cup J_{r_{n}}$ for every $n \in \mathbb{N}$. Note that

$$
|\mu|\left(Y \backslash K_{n}\right) \leq \frac{\epsilon}{2^{n}},
$$

and the restriction of $\gamma_{n}$ to $K_{n}$ is continuous.

Define $K=\bigcap_{n \in \mathbb{N}} K_{n}$. Observe that $|\mu|(Y \backslash K) \leq \epsilon$ and the sequence $\left(\gamma_{n}\right)_{n \in \mathbb{N}}$, restricted to $K$, converges uniformly to $\gamma$ on the metric d. Hence, the restriction of $\gamma$ to $K$ is continuous with respect to the weak ${ }^{*}$-topology of $E^{*}$.

(b) In this case the function $\gamma$ is a pointwise limit of a sequence $\left(\gamma_{n}\right)_{n \in \mathbb{N}}$ of simple functions. Given $\epsilon>0$, the Egoroff Theorem [11, Theorem 42, p. 18] produces a measurable set $A \subset Y$ such that $|\mu|(A) \leq \frac{\epsilon}{2}$, and the sequence $\left(\gamma_{n}\right)_{n \in \mathbb{N}}$, restricted to $Y \backslash A$, converges uniformly to $\gamma$ in norm. Working with functions $\gamma_{n}$ similarly as in the previous case, we may find a compact $K \subset Y \backslash A$ with the required properties. 


\section{ON LOWER-SEMICONTINUOUS FUNCTIONS}

Next we introduce some lower-semicontinuous functions [20, Definition 6.3.1, p. 97] which will be used in the sequel.

Lemma 4.1. Let $E$ be a Banach space and $X$ and $Y$ be locally compact Hausdorff spaces. Suppose that $T$ is a bounded linear operator from $C_{0}(X, E)$ to $C_{0}(Y, E)$ and $\gamma: Y \rightarrow E^{*}$ is bounded and continuous in the weak $k^{*}$-topology of $E^{*}$. Then for every open set $U \subset X$ the application

$$
y \longmapsto\left|T^{*}\left(\gamma(y) \delta_{y}\right)\right|(U)
$$

is lower-semicontinuous.

Proof. For each $f \in C_{0}(X, E)$ the application

$$
y \longmapsto\left|\int f d T^{*}\left(\gamma(y) \delta_{y}\right)\right|=|\langle\gamma(y),(T f)(y)\rangle|
$$

is continuous, since it is a composition of continuous functions. Then, by considering $\mathcal{F}=\left\{f \in C_{0}(X, E):\|f\| \leq 1\right.$ and $f(x)=0$ for every $\left.x \in X \backslash U\right\}$,

$$
y \longmapsto\left|T^{*}\left(\gamma(y) \delta_{y}\right)\right|(U)=\sup _{\mathcal{F}}\left|\int f d T^{*}\left(\gamma(y) \delta_{y}\right)\right|
$$

is lower-semicontinuous.

We end this short section by summarizing in the next proposition a result about lower-semicontinuous functions and $r c a b v(X)$ spaces which was established in the proof of the main theorem of [7].

Proposition 4.2. Let $X$ and $Y$ be locally compact Hausdorff spaces. Fix $x \in X$ and assume that $\left\{\varphi_{y}: y \in Y\right\}$ is a family of positive measures in rcabv $(X)$ such that $\varphi_{y}(\{x\})=0$ for every $y \in Y$. Suppose that $\left\{U_{i}: i \in I\right\}$ is the collection of all open neighborhoods of $x$ endowed with the partial ordering of reverse inclusion.

Pick $\epsilon>0$. For every $i \in I$ consider the following sets:

$$
C_{i}=\overline{\left\{y \in Y: \varphi_{y}\left(\overline{U_{i}}\right) \leq \epsilon\right\}} .
$$

If for every open set $U \subset Y$ the application $y \mapsto \varphi_{y}(U)$ is lower-semicontinuous, then for every positive measure $\mu \in \operatorname{rcabv}(Y)$ we have

$$
\mu(Y)=\sup _{i \in I} \mu\left(C_{i}\right) .
$$

\section{Proof of Theorem 1.5}

We are now ready to prove the main theorem of this work.

Proof. First of all notice that thanks to Lemma 2.2 we can assume that $X$ and $Y$ are infinite. We will prove that $|X| \leq|Y|$. Thus by symmetry $|Y| \leq|X|$, and we are done.

We will apply Proposition 2.1 (b) to prove $|X| \leq|Y|$. So, it is enough to show that for every $x \in X$ there exists a $y \in Y$ such that $x \in X_{y}$. By contradiction, suppose that there exists a $x \in X$ such that, for each $y \in Y$ and for each $\varphi \in S_{E}^{*}$,

$$
\left|T^{*}\left(\varphi \delta_{y}\right)\right|(\{x\})=\left\|T^{*}\left(\varphi \delta_{y}\right)(\{x\})\right\|=0 .
$$

Fix $\Phi \in S_{E}^{*}$ and consider $\mu_{x} \in \operatorname{rcabv}\left(Y, E^{*}\right)$ defined by

$$
\mu_{x}=T^{-1^{*}}\left(\Phi \delta_{x}\right) \text {. }
$$


By Theorem 3.1 and Remark 3.2 there exists a weakly*-measurable function $\gamma: Y \rightarrow E^{*}$ verifying the statements of this theorem which is measurable in the case where $E^{*}$ has the Radon-Nikodým property.

Pick $\epsilon>0$ such that

$$
\epsilon<\min \left\{\left|\mu_{x}\right|(Y), \frac{1}{2\left(\|T\|+\left|\mu_{x}\right|(Y)\right)}\right\} .
$$

According to Proposition 3.3, there is a compact $K \subseteq Y$ such that

$$
\left|\mu_{x}\right|(Y \backslash K) \leq \epsilon
$$

and the restriction of $\gamma$ to $K$ is continuous with respect to the weak*-topology of $E^{*}$.

For each $y \in K$, write

$$
\Gamma_{y}=\left|T^{*}\left(\gamma(y) \delta_{y}\right)\right| .
$$

Thus, it follows from (5.1) that $\Gamma_{y}(\{x\})=0$, for each $y \in K$.

Now, let $\left\{U_{i}: i \in I\right\}$ be the collection of all open neighborhoods of $x$ endowed with the partial ordering of reverse inclusion. For each $i \in I$, define the following measurable set:

$$
C_{i}=\overline{\left\{y \in K: \Gamma_{y}\left(\overline{U_{i}}\right) \leq \epsilon\right\}} .
$$

Moreover, by Lemma 4.1, notice that for every open set $U \subset X$ the application $y \mapsto \Gamma_{y}(U)$ is lower-semicontinuous. Therefore by Proposition 4.2 we have

$$
\left|\mu_{x}\right|(K)=\sup _{i \in I}\left|\mu_{x}\right|\left(C_{i}\right) .
$$

Let $v \in E$ be such that $\|v\|=1$ and $\frac{1}{2}<\langle\Phi, v\rangle$. By the Urysohn Lemma, for each $i \in I$ there is $h_{i} \in C_{0}(X)$ with $0 \leq h_{i} \leq 1, h_{i}(x)=1$ and $h_{i}(z)=0$ if $z \in X \backslash U_{i}$. Now define $f_{i} \in C_{0}(X, E)$ by $f_{i}=v \cdot h_{i}$. Hence by (5.2),

$$
\frac{1}{2}<\left\langle\Phi, f_{i}(x)\right\rangle=\int f_{i} d\left(\Phi \delta_{x}\right)=\int T f_{i} d\left(T^{-1^{*}}\left(\Phi \delta_{x}\right)\right)=\int\left(T f_{i}\right) d \mu_{x} .
$$

Since every $f \in C_{0}(Y, E)$ is a uniform limit of a sequence of $E$-valued simple functions, it follows from Theorem 3.1 (b) that

$$
\int\left(T f_{i}\right) d \mu_{x}=\int\left\langle\gamma(y),\left(T f_{i}\right)(y)\right\rangle d\left|\mu_{x}\right|(y),
$$

for each $i \in I$. We will split this integral into three parts. In order to do this, first observe that, in view of (5.4) and Theorem 3.1 (a),

$$
\left|\int_{Y \backslash K}\left\langle\gamma(y),\left(T f_{i}\right)(y)\right\rangle d\right| \mu_{x}|(y)| \leq\|T\|\left|\mu_{x}\right|(Y \backslash K) \leq\|T\| \epsilon .
$$

On the other hand, for every $y \in K$ and for each $i \in I$, by (5.5) we infer that

$$
\left|\left\langle\gamma(y),\left(T f_{i}\right)(y)\right\rangle\right|=\left|\int f_{i} d T^{*}\left(\gamma(y) \delta_{y}\right)\right| \leq \Gamma_{y}\left(\overline{U_{i}}\right) .
$$

But, by using the fact that the restriction of $\gamma$ to $K$ is continuous with respect to the weak*-topology of $E^{*}$, if $y \in C_{i}$, then according to (5.6) we deduce that $\left|\left\langle\gamma(y),\left(T f_{i}\right)(y)\right\rangle\right| \leq \epsilon$. Then we have

$$
\left|\int_{C_{i}}\left\langle\gamma(y),\left(T f_{i}\right)(y)\right\rangle d\right| \mu_{x}|(y)| \leq \epsilon\left|\mu_{x}\right|(Y) .
$$


So, putting (5.10) and (5.11) together, by Theorem 3.1 (a) we obtain

$$
\begin{aligned}
\int\left\langle\gamma(y),\left(T f_{i}\right)(y)\right\rangle d\left|\mu_{x}\right|(y) & \leq \epsilon\|T\|+\left|\int_{K \backslash C_{i}}\left\langle\gamma(y),\left(T f_{i}\right)(y)\right\rangle d\right| \mu_{x}|(y)|+\epsilon\left|\mu_{x}\right|(Y) \\
& \leq \epsilon\left(\|T\|+\left|\mu_{x}\right|(Y)\right)+\|T\|\left(\left|\mu_{x}\right|(K)-\left|\mu_{x}\right|\left(C_{i}\right)\right) .
\end{aligned}
$$

Furthermore, keeping in mind (5.7), (5.8), (5.9) and the choice of $\epsilon$ in (5.3), we conclude that

$$
\frac{1}{2} \leq \epsilon\left(\|T\|+\left|\mu_{x}\right|(Y)\right)<\frac{1}{2}
$$

a contradiction which completes the proof of Theorem 1.5

\section{REFERENCES}

[1] D. Amir, On isomorphisms of continuous function spaces. Israel J. Math., 3, (1965), 205-210. MR0200708 (34:596)

[2] S. Banach, Théorie des opérations linéaires. Monografie Matematyczne, Warsaw, 1933.

[3] E. Behrends and M. Cambern, An isomorphic Banach-Stone theorem. Studia Math., 90, (1988), 15-26. MR947918 (89h:46020)

[4] M. Cambern, Isomorphisms of $C_{0}(Y)$ onto $C(X)$. Pacific J. Math., 35, (1970), 307-312. MR0433201 (55:6180)

[5] M. Cambern, Isomorphims of spaces of continuous vector-valued functions. Illinois J. Math., 20, (1976), 1-11. MR0388078 (52:8915)

[6] M. Cambern, On isomorphisms with small bound. Proc. Amer. Math. Soc., 18, (1967), 10621066. MR0217580 (36:669)

[7] B. Cengiz, On topological isomorphisms of $C_{0}(X)$ and the cardinal number of $X$. Proc. Amer. Math. Soc., 72, 1, (1978), 105-108. MR0493291 (58:12319)

[8] H. B. Cohen, A bound-two isomorphism between $C(X)$ Banach spaces. Proc. Amer. Math. Soc., 50, 1, (1975), 215-217. MR0380379 (52:1279)

[9] J. Diestel and J.J. Uhl, Jr., Vector Measures. Math. Surveys 15, Amer. Math. Soc., Providence, R.I., 1977. MR0453964(56:12216)

[10] J. Diestel, H. Jarchow, A. Tonge, Absolutely Summing Operators. Cambridge Studies in Advanced Mathematics, 43. Cambridge University Press, Cambridge, 1995. MR.1342297 (96i:46001)

[11] N. Dinculeanu, Vector Integration and Stochastic Integration in Banach Spaces. Wiley Interscience, 2000. MR1782432 (2001h:60096)

[12] P. Enflo, Banach spaces which can be given an equivalent uniformly convex norm. Israel J. Math., 13, 3-4, (1972), 281-288. MR0336297 (49:1073)

[13] R. Engelking, General Topology. Sigma Series in Pure Mathematics, vol. 6, HeldermannVerlag, Berlin, 1989. MR1039321 (91c:54001)

[14] W. Hensgen, A simple proof of Singer's representation theorem. Proc. Amer. Math. Soc., 124, 10, (1996), 3211-3212. MR1343697 (96m:46061)

[15] K. Jarosz, Small isomorphisms of $C(X, E)$ spaces. Pacific J. Math., 138, 2, (1989), 295-315. MR.996203 (90f:46056)

[16] W. B. Johnson, J. Lindenstrauss, Basics concepts in the geometry of Banach spaces. Handbook of the geometry of Banach spaces. North-Holland Publishing Co., Amsterdam, 2001, 1-84. MR.1863689 (2003f:46013)

[17] L. Meziani, On the dual space $C_{0}^{*}(S, X)$. Acta Math. Univ. Comenianae., LXXVIII, 1 , (2009), 153-160. MR2684182 (2011g:46069)

[18] H. P. Rosenthal, The Banach space $C(K)$. Handbook of the geometry of Banach spaces. North-Holland Publishing Co., Amsterdam, 2001, 1547-1602. MR1999603 (2004g:46028)

[19] C. Samuel, Sur la reproductibilite des espaces $l_{p}$. Math. Scand., 45, (1979), 103-117. MR.567436 (81e:46062) 
[20] Z. Semadeni, Banach Spaces of Continuous Functions Vol. I. Monografie Matematyczne, Tom 55. Warsaw, PWN-Polish Scientific Publishers, Warsaw, 1971. MR0296671 (45:5730)

[21] M. H. Stone, Applications of the theory of Boolean rings to general topology. Trans. Amer. Math. Soc., 41, (1937), 375-481. MR.1501905

Department of Mathematics, University of São Paulo, ime, Rua do Matão 1010, São PAUlo, BraziL

E-mail address: lc@ime.usp.br

Department of Mathematics, University of São Paulo, ime, Rua do Matão 1010, São Paulo, Brazil

E-mail address: eloi@ime.usp.br 